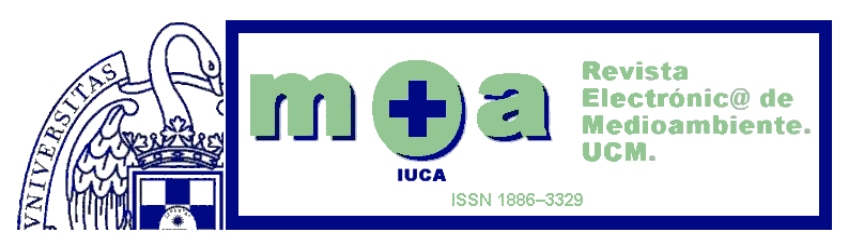

\title{
PERCEPCIÓN AMBIENTAL EN DOS COMUNIDADES CUBANAS
}

\author{
María Borroto Pérez \\ Profesor Investigador de la Universidad de Ciego de Ávila, Cuba \\ mariaborroto@gmail.com \\ Lucas Rodríguez Pérez \\ Profesor Investigador de la Universidad de Ciego de Ávila, Cuba \\ pfa_lucas@agronomia.unica.cu \\ Aurelio Reyes Ramírez \\ Profesor investigador Universidad Autónoma Chapingo, México \\ aueiorey@yahoo.com.mx
}

\author{
Blanca Alejandra López Vázquez \\ Profesora foránea de educación primaria, Tezoyuca, México \\ blancaale2003@yahoo.com.mx
}

Recibido: 21 de diciembre de 2010

Aceptado: 4 de abril de 2011

\section{RESUMEN}

Una parte considerable de nuestro desempeño depende de la capacidad para percibir adecuadamente los diferentes elementos que conforman el medio; nuestras percepciones ambientales condicionan las actitudes, sensibilidades, orientación y regulación de las acciones hacia el entorno. Como parte de un proyecto investigativo se evaluó la percepción ambiental de los miembros de las comunidades organizados en cuatro grupos meta. Se diseñó y validó por expertos un cuestionario y cuatro variables para utilizarlo de base en encuestas a la población de dichas comunidades. Se consideraron las variables visión ambiental, responsabilidad ambiental, actitud ambiental y decisión a incorporarse al cambio y visión sobre la preparación ambiental. Se diagnosticó la percepción ambiental de cada uno de los grupos meta en las dos comunidades, encontrando la variable mejor evaluada la formación, seguida de la actitud y visión ambientales. Esto permitió la implementación de una estrategia contextualizada para la educación ambiental.

Palabras clave: Percepción ambiental, diagnóstico, visión ambiental.

\section{ENVIRONMENTAL PERCEPTION IN TWO CUBAN COMMUNITIES}

\begin{abstract}
A considerable part of our newspaper acting depends on the capacity to perceive the different elements that conform the means in which unwrap ourselves, our environmental perceptions appropriately they condition the attitudes, sensibilities, and they influence considerably in the orientation and regulation of our actions toward the environment; it is for it that is important the study of this process, by way of to contribute to that the human development is adapted and harmonious, according to the characteristics of the environment that obtain a coherent behavior with the pattern of the sustainable. As part of an investigative project the environmental perception of the
\end{abstract}


members of the communities was evaluated organized in four goal groups. It was designed and it validated for experts a questionnaire and four variables to use it of base in surveys to the population of this communities. They were considered the variable environmental vision, environmental responsibility, environmental attitude and decision to incorporate to the change and vision on the environmental preparation. The environmental perception was diagnosed of each one of the groups goal in the two communities, finding the evaluated better variable the formation, followed by the attitude and environmental vision. This will allow the design and implementation of a strategy in the context for the environmental education and with it knowledge, attitudes and consequent environmental behavior with the sustainable pattern of development.

Keywords: Environmental, diagnostic perception, environmental vision.

\section{UNE PERCEPTION ENVIRONNEMENTALE DANS DEUX COMMUNAUTES CUBAINES}

\section{RESUMÉ}

Une partie considérable de notre performance dépend de la capacité de bien percevoir les différents éléments qui composent le milieu, de notre condition environnementale perceptions des attitudes, des sensibilités, d'orientation et régulation des actions envers l'environnement. Dans le cadre d'un projet de recherche évalue la perception de l'environnement des membres de la communauté organisée en quatre groupes cibles. A été conçu et validé par des experts à un questionnaire et quatre variables de base pour une utilisation dans les enquêtes sur la population de ces communautés. Les variables ont été considérées comme vision de l'environnement, responsabilité environnementale, l'attitude de l'environnement et la détermination à se joindre à la vision du changement et le développement de l'environnement. la perception de l'environnement a été diagnostiqué dans chacun des groupes cibles dans les deux communautés, de trouver la meilleure formation variable évaluée, suivie par l'attitude de l'environnement et de la vision. Cela a permis la mise en œuvre d'une stratégie pour l'éducation environnementale dans le contexte.

Mots-clés: la perception de l'environnement, le diagnostic, la vision de l'environnement.

\section{INTRODUCCIÓN}

A partir de la década del 70 en el mundo se comienza a tratar la cuestión ambiental debido al crecimiento y evidente deterioro del entorno, cuya causa fundamental ha sido la acción del hombre, que pone en peligro su propia sobrevivencia y perpetuación como especia biológica.

Se hace necesario promover la formación de una conciencia ambiental adecuada, que les permita convivir con el entorno, preservarlo y transformarlo en función de sus necesidades sin comprometer con ello las posibilidades de las generaciones futuras de satisfacer las suyas (Alea, 2005:1)

Para ello se necesita llevar a cabo la educación ambiental que se concibe como un proceso permanente en el que los individuos y la colectividad toman conciencia de su entorno y adquieren los conocimientos, los valores, las concepciones, la experiencia y la voluntad que les permitirá actuar individual y colectivamente para resolver los problemas 
actuales y futuros del medio ambiente. Se considera un modelo teórico, metodológico y práctico que trasciende el sistema educativo tradicional y alcanza la concepción de medio ambiente y de desarrollo (CITMA, 1997:27). Es un proceso educativo para cada individuo, las familias, las comunidades, la sociedad y el Estado, para tomar decisiones y actuar en pro del desarrollo sostenible mundial.

Pero antes debe ser comprendida la problemática ambiental, es preciso conocer cuáles son las percepciones ambientales de los individuos y colectivos que componen una comunidad dada, ya que ellas condicionan las actitudes, las sensibilidades e influyen considerablemente en la orientación y regulación de las acciones hacia el entorno.

La percepción "consiste en el reflejo en la conciencia del hombre de los objetos o fenómenos, al actuar directamente sobre los sentidos, durante cuyo proceso ocurren la regulación y unificación de las sensaciones aisladas en reflejos integrales de cosas y acontecimientos" (Alea, 2005:5). La percepción ambiental considera el entorno de forma holística y para ello las investigaciones que la tratan tienen su foco de atención en el estudio de las múltiples experiencias ambientales que una persona puede tener en su relación con el entorno. Colmenares (2001:7) señala la necesidad de profundizar en la construcción de indicadores rigurosos sobre la "percepción" ambiental.

De allí que el objetivo del presente trabajo haya sido evaluar la percepción ambiental de diferentes grupos meta de las comunidades Cacahual Nuevo y CPA Ignacio Agramonte como punto de partida para el desarrollo de un proyecto para la educación ambiental de sus miembros.

\section{MATERIALES Y MÉTODOS}

Las dos comunidades están ubicadas en el Municipio Ciro Redondo; Cacahual Nuevo es clasificada como semiurbana, mientras que la Cooperativa de producción agropecuaria (CPA) Ignacio Agramonte es una comunidad rural. La población y los grupos meta se muestran en la tabla 1.

Tabla 1 Número de habitantes por grupos meta de las comunidades estudiadas

\begin{tabular}{|l|c|c|c|c|}
\hline Grupos meta seleccionados & \multicolumn{4}{c|}{ Número de habitantes } \\
\hline & \multicolumn{1}{|c|}{ Cacahual Nuevo } & CPA Ignacio Agramonte \\
\hline & población & muestra & población & muestra \\
\hline Directivos (D) & 15 & 15 & 7 & 7 \\
\hline Trabajadores y campesinos (T-C) & 385 & 204 & 65 & 58 \\
\hline Amas de casa y jubilados (A-J) & 173 & 143 & 42 & 38 \\
\hline Estudiantes (E) & 113 & 80 & 20 & 20 \\
\hline Población total & 1039 & 442 & 134 & 123 \\
\hline
\end{tabular}

Se caracterizaron las comunidades en tres grupos: por su edad (menos de 35, entre 35 y 50 y más de 50 años), por su nivel escolar (estudios primarios, secundarios, preuniversitarios y universitarios) y por el tiempo que lleva viviendo en la comunidad (menos de 10 años, entre 10 y 20 y más de 20 ). Los resultados se muestran en la tabla 2.

En la CPA la muestra es más o menos homogénea por grupo de edades; el mayor número de miembros son los de nivel preuniversitario y los que viven en la comunidad por más de 20 años. En Cacahual Nuevo existen más jóvenes, predominan los de nivel secundario y preuniversitario y también los que viven hace más de 20 años allí. 
Tabla 2 caracterización de la muestra por grupos

\begin{tabular}{|l|c|c|}
\hline Grupos & Cacahual & CPA \\
\hline Menos de 35 años & 179 & 42 \\
\hline Entre 35 y 50 años & 120 & 45 \\
\hline Más de 50 años & 143 & 36 \\
\hline Analfabeto o nivel primario & 75 & 20 \\
\hline Nivel secundario & 150 & 35 \\
\hline Preuniversitario & 152 & 54 \\
\hline Universitario & 65 & 14 \\
\hline Menos de 10 años viviendo & 51 & 21 \\
\hline Entre 10 y 20 años & 110 & 33 \\
\hline Más de 20 años & 281 & 60 \\
\hline
\end{tabular}

Para evaluar la percepción ambiental de la comunidad fue necesario un profundo análisis y revisión bibliográfica previa y posterior consulta con expertos a partir de lo cual se definieron la percepción ambiental y cuatro de sus variables, que permiten evaluarla: La Visión ambiental y de los problemas ambientales, la Responsabilidad ambiental, la Actitud ambiental y decisión a incorporarse al cambio y la Formación medioambiental, las que se definieron como sigue.

Percepción ambiental: capacidad para percibir adecuadamente los diferentes elementos que conforman el medio en el cual nos desenvolvemos, que condicionan las actitudes, sensibilidades, e influyen considerablemente en la orientación y regulación de nuestras acciones hacia el entorno

\section{Variables:}

Visión del medio ambiente y de los problemas ambientales Idea que tiene el individuo sobre el medio ambiente y sus dimensiones, así como de los problemas ambientales y su extensión

Responsabilidad ambiental Apreciación que tienen los individuos sobre los agentes causantes de los problemas ambientales, así como los que deben intervenir para evitarla

\section{Actitud ambiental y decisión a incorporarse al cambio}

predisposición del pensamiento humano a actuar a favor o en contra del entorno social, teniendo como base las vivencias, los conocimientos y los valores del individuo con respecto a su entorno, que proyectan en una dirección determinada y poseen un nivel de intensidad (fuerte o débil).

\section{Formación medioambiental}

Impresión que tienen los individuos sobre la calidad y efectividad de las acciones de educación ambiental que han recibido así como los Modos y medios mediante los cuales la han adquirido

Se sometieron a validación de expertos la representatividad, la coherencia y la calidad técnica de las definiciones teórica y operacional, ítems y criterios de evaluación de cada variable, de acuerdo con criterios de Barraza (2007:12), obteniéndose una validación fuerte en todos ellos, tal como se representa en el anexo 1.

A partir de aquí se construyó el cuestionario para aplicar a los diferentes grupos meta. (anexo 2).

Antes de aplicar las encuestas a la muestra completa se evaluó la fiabilidad de la misma, aplicada al $20 \%$ de dicha muestra, mediante el Alfa de Cronbach, con los resultados que se muestran en la tabla 3. 
Tabla 3 Análisis de fiabilidad. Resumen del procesamiento de los casos

\begin{tabular}{|cl|c|c|c|}
\hline & $\mathrm{N}$ & $\%$ & Alfa de Cronbach \\
\hline Casos & Válidos & 42 & 71,2 & 0,942 \\
& Excluidos(a) & 17 & 28,8 & \\
Total & 59 & 100,0 & \\
\hline
\end{tabular}

a Eliminación por lista basada en todas las variables del procedimiento.

Se seleccionó una muestra representativa de la población de cada uno de los grupos meta mediante la fórmula $n=N /\left[(e)^{2}(N-1)+1\right]$, obteniéndose los datos que se exponen en la tabla 1.

Todos los análisis estadísticos se basaron en la utilización del paquete profesional SPSS v.15.00 para Windows 2006. Se realizó la prueba no paramétrica de Kruskal Wallis y el ANOVA.

\section{RESULTADOS Y DISCUSIÓN}

Para evaluar la percepción ambiental se exponen los resultados obtenidos por variables y grupos meta en cada una de las comunidades. Se tuvo en cuanta esto por coincidir con los criterios expuestos en el trabajo Elementos Básicos de percepción ambiental (2008:1) donde se considera que en su percepción ambiental la persona organiza su experiencia en el entorno a partir de unos determinados propósitos u objetivos, ques pueden ir desde objetivos esencialmente utilitaristas o funcionalistas hasta objetivos de carácter emocional, estético o relacional.

Visión del medio ambiente y de los problemas ambientales

En la Tabla 4.1 aparecen los resultados de la evaluación de la visión ambiental de los integrantes de las dos comunidades. (Muy amplia ó 5, amplia ó 4, media ó 3, estrecha ó 2 y muy estrecha ó 1 ). En la tabla 4.2 se expresan las evaluaciones obtenidas por grupos de edad, nivel escolar, tiempo de vida en la comunidad y ocupación, con el correspondiente análisis estadístico.

Tabla 4.1 Número de miembros evaluados en la Visión ambiental

\begin{tabular}{|c|c|c|c|c|c|c|c|c|c|c|}
\hline \multirow{3}{*}{$\begin{array}{l}\text { Grupo } \\
\text { meta }\end{array}$} & \multicolumn{10}{|c|}{ Evaluación } \\
\hline & \multicolumn{2}{|c|}{ MA (5) } & \multicolumn{2}{|c|}{ A (4) } & \multicolumn{2}{|c|}{ M (3) } & \multicolumn{2}{|c|}{$E(2)$} & \multicolumn{2}{|c|}{ ME (1) } \\
\hline & C.Nvo & CPA & C.Nvo & CPA & C.Nvo & CPA & C.Nvo & CPA & C.Nvo & CPA \\
\hline$D$ & 0 & 0 & 7 & 2 & 7 & 4 & 1 & 1 & 0 & 0 \\
\hline$A-J$ & 0 & 0 & 39 & 8 & 63 & 19 & 32 & 7 & 9 & 4 \\
\hline$E$ & 0 & 0 & 17 & 7 & 51 & 8 & 8 & 5 & 4 & 0 \\
\hline $\mathrm{T}-\mathrm{C}$ & 0 & 1 & 41 & 22 & 126 & 17 & 25 & 12 & 12 & 6 \\
\hline total & 0 & 1 & 104 & 39 & 247 & 48 & 66 & 25 & 25 & 10 \\
\hline
\end{tabular}




\begin{tabular}{|c|c|c|c|}
\hline Variable dependient & visión & Medias & Sig. \\
\hline $\begin{array}{l}\text { Cacahual } \\
\text { CPA }\end{array}$ & $\begin{array}{l}\text { menos de } 35 \text { años } \\
\text { Entre } 36 \text { y } 50 \\
\text { más de } 50 \\
\text { menos de } 35 \\
\text { Entre } 36 \text { y } 50 \\
\text { más de } 50\end{array}$ & $\begin{array}{l}2.98 \\
3.05 \\
2.91 \\
2,97 \\
3,04 \\
2,81\end{array}$ & NS \\
\hline $\begin{array}{l}\text { Nivel escolar } \\
\text { Cacahual }\end{array}$ & $\begin{array}{l}\text { primario } \\
\text { secundario } \\
\text { preuniversitario } \\
\text { universitarios } \\
\text { primario } \\
\text { secundario } \\
\text { preuniversitario } \\
\text { universitarios }\end{array}$ & $\begin{array}{l}2.77 \\
2.97 \\
3.01 \\
3.12 \\
2,73 \\
2,71 \\
2,98 \\
3,67\end{array}$ & $\begin{array}{l}\mathrm{b} \\
\mathrm{ab} \\
\mathrm{a} \\
\mathrm{a} \\
\mathrm{b} \\
\mathrm{b} \\
\mathrm{b} \\
\mathrm{a}\end{array}$ \\
\hline $\begin{array}{l}\text { Tiempo de vida allí } \\
\text { Cacahual } \\
\text { CPA }\end{array}$ & $\begin{array}{l}\text { menos de } 10 \text { años } \\
\text { entre } 11 \text { y } 20 \\
\text { mas de } 20 \\
\text { menos de } 10 \text { años } \\
\text { entre } 11 \text { y } 20 \\
\text { mas de } 20\end{array}$ & $\begin{array}{l}2.94 \\
2.97 \\
2.99 \\
2,93 \\
2,94 \\
2,97\end{array}$ & NS \\
\hline Ocupación Cacahual & $\begin{array}{l}\text { directivos } \\
\text { estudiantes } \\
\text { trabajadores y campesinos } \\
\text { Amas de casa y jubilados } \\
\text { directivos } \\
\text { estudiantes } \\
\text { trabajadores y campesinos } \\
\text { Amas de casa y jubilados }\end{array}$ & $\begin{array}{l}3.40 \\
3.01 \\
2.97 \\
2.93 \\
3,14 \\
3,10 \\
2,97 \\
2,81\end{array}$ & $\begin{array}{l}a \\
b \\
b \\
b \\
N S\end{array}$ \\
\hline
\end{tabular}

El $70,7 \%$ de la CPA y el $79,4 \%$ de Cacahual tienen una visión del medio ambiente y de los problemas ambientales entre media y alta, lo cual es favorable ya que puede indicar que sus ideas sobre el medio ambiente y sus dimensiones, así como de los problemas ambientales y su extensión son adecuadas. Si se analiza por grupos meta se observa que los de mejor visión ambiental en Cacahual son los directivos (93,3\% entre media y alta) seguidos de los estudiantes $(63,7 \%$ entre $A$ y $M)$ y de los trabajadores y campesinos $(61,8 \%)$. Sólo el $44 \%$ de las amas de casa y los jubilados obtuvieron una visión evaluada de media.

Si se hace un análisis estadístico de estos resultados, los directivos tuvieron una visión significativamente superior al resto de las ocupaciones en Cacahual, mientras que en la CPA no se manifestaron diferencias significativas.

Si tenemos en cuenta el nivel escolar se observa que en Cacahual todos los directivos, el $62,2 \%$ de los estudiantes y el $57,3 \%$ de los trabajadores y campesinos tienen un nivel de preuniversitario o universidad. Sin embargo sólo el $24,5 \%$ de las 
amas de casa y jubilados alcanzan dicho nivel. Entonces puede ser éste uno de los factores que han influido en este resultado.

En la CPA la situación fue diferente. Los de mayor visión resultaron ser los estudiantes (75\% A y $M$ ), seguidos de los trabajadores y campesinos (67,2\% A y M) y los directivos $(57,1 \% \mathrm{M})$. Sólo el $50 \%$ de las amas de casa y jubilados obtuvo una evaluación media de su visión.

Si se hace un análisis estadístico de estos resultados el incremento del nivel escolar elevó la visión de forma significativa en Cacahual y en la CPA los universitarios tuvieron una visión significativamente superior al resto de los habitantes.

En general entre los grupos meta no se observó una influencia marcada de la edad y tiempo de vida en la comunidad, tampoco en el análisis estadístico.

Al identificar los elementos que conforman el medio ambiente en las encuestas se observó, como tendencia, la exclusión de la dimensión social del mismo, al igual que los problemas ambientales de esta dimensión.

La mayoría reconocía que los problemas ambientales estaban extendidos a todo el planeta. En cuanto a la importancia que le atribuyen, las respuestas fueron diversas desde un peligro inminente hasta que el tema está de moda.

Estos resultados son similares a los obtenidos por CIGEA (1999:1) donde se encontró que la amplia mayoría tiene una concepción restringida $(75 \%)$ y un pequeño segmento expresa una concepción amplia (22\%). No apreciaron una relación con la edad pero si con el nivel de escolaridad y de los que se encuentran vinculados a una institución estatal.

Por otra parte Colmenares (2001:2) encontró que la mayoría de los entrevistados poseía una concepción reduccionista del ambiente: Tan sólo el $21 \%$ incluyó la dimensión social como un componente del medio ambiente Este autor considera que el hecho de que un numeroso grupo de encuestados tenga una visión estrecha de lo que es el medio ambiente, sugiere que para muchos la naturaleza continúa siendo el depósito de materias primas del que el hombre hace uso y, del que con frecuencia abusa.

\section{Responsabilidad ambiental}

En la Tabla 5.1 aparecen los resultados de la evaluación de la Responsabilidad ambiental de los integrantes de las dos comunidades. (Excelente ó 5, muy bien ó 4, Bien ó 3, Regular ó 2 y mal ó 1). En la tabla 5.2 se expresan las evaluaciones obtenidas por grupos de edad, nivel escolar, tiempo de vida en la comunidad y ocupación, con el correspondiente análisis estadístico.

Tabla 5.1 Número de miembros evaluados en la Responsabilidad ambiental

\begin{tabular}{|l|c|c|c|c|c|c|c|c|c|c|}
\hline $\begin{array}{l}\text { Grupo } \\
\text { meta }\end{array}$ & \multicolumn{9}{|c|}{ Evaluación } \\
\hline & \multicolumn{1}{|c|}{ E (5) } & \multicolumn{2}{c|}{ MB (4) } & \multicolumn{2}{c|}{ B (3) } & \multicolumn{2}{c|}{ R (2) } & \multicolumn{2}{c|}{ M (1) } \\
\hline & C.Nvo & CPA & C.Nvo & CPA & C.Nvo & CPA & C.Nvo & CPA & C.Nvo & CPA \\
\hline D & 0 & 0 & 0 & 0 & 1 & 0 & 12 & 5 & 2 & 2 \\
\hline A-J & 0 & 0 & 4 & 0 & 4 & 0 & 57 & 0 & 78 & 38 \\
\hline E & 0 & 0 & 0 & 0 & 0 & 0 & 29 & 2 & 51 & 18 \\
\hline T-C & 0 & 0 & 0 & 0 & 26 & 1 & 73 & 23 & 105 & 34 \\
\hline total & 1 & 0 & 4 & 0 & 30 & 2 & 171 & 30 & 236 & 92 \\
\hline
\end{tabular}


Tabla 5.2 Evaluación de la responsabilidad ambiental por grupos y su significación estadística

\begin{tabular}{|c|c|c|c|}
\hline \multicolumn{2}{|c|}{ Variable dependiente Responsabilidad } & Medias & Sig. \\
\hline \multirow[t]{3}{*}{ Cacahual } & menos de 35 años & 2.44 & \\
\hline & Entre 36 y 50 & 2.52 & a \\
\hline & más de 50 & 2.29 & $b$ \\
\hline \multirow[t]{3}{*}{ CPA } & menos de 35 & 2,61 & a \\
\hline & Entre 36 y 50 & 2,54 & a \\
\hline & más de 50 & 2,03 & $b$ \\
\hline \multirow{8}{*}{$\begin{array}{l}\text { Nivel escolar } \\
\text { Cacahual }\end{array}$} & nrimario & 2.10 & $b$ \\
\hline & secundario & 2.44 & a \\
\hline & preuniversitario & 2.53 & a \\
\hline & universitarios & 2.45 & a \\
\hline & primario & & \\
\hline & secundario & 1.96 & c \\
\hline & preuniversitario & 2.23 & bc \\
\hline & universitarios & 2.60 & $a b$ \\
\hline \multirow{6}{*}{$\begin{array}{l}\text { Tiempo de vida allí } \\
\text { Cacahual }\end{array}$} & menos de 10 años & 2.80 & a \\
\hline & entre 11 y 20 & 2.56 & NS \\
\hline & mas de 20 & 2.47 & \\
\hline & menos de 10 años & 2.37 & \\
\hline & entre 11 y 20 & 2,81 & $\mathrm{a}$ \\
\hline & mas de 20 & 2,15 & $b$ \\
\hline \multirow[t]{8}{*}{ Ocupación $\quad$ Cacahual } & directivos & 2,35 & b \\
\hline & estudiantes & 3.13 & a \\
\hline & trabajadores y campesinos & 2.31 & $b$ \\
\hline & Amas de casa y jubilados & 2.49 & b \\
\hline & directivos & 2.29 & b \\
\hline & estudiantes & 3,00 & $d$ \\
\hline & trabajadores y campesinos & 2,60 & a \\
\hline & Amas de casa y jubilados & 2,48 & $a b$ \\
\hline
\end{tabular}

La percepción que tienen los miembros de la comunidad sobre la responsabilidad en la conservación o agresión al medio ambiente resultó evaluada de mal para el 74,7\% de los pobladores de la CPA y de R y M para el 92,1\% de Cacahual. Esto se comportó de manera similar en un análisis por grupos meta, donde todas las amas de casa, jubilados y directivos, el $90 \%$ de los estudiantes y el $98,3 \%$ de los trabajadores y campesinos de la CPA obtuvieron esa evaluación. En Cacahual la situación fue algo más favorable ya que el $80 \%$ de los directivos fue de $\mathrm{R}$, el $40 \%$ de las amas de casa fue $\mathrm{R}$, pero el $63,7 \%$ de los estudiantes y el $51,5 \%$ de los trabajadores fueron evaluados de mal.

Si se hace un análisis estadístico de estos resultados en Cacahual los directivos tuvieron una responsabilidad ambiental significativamente superior y en la CPA, sólo las amas de casa y jubilados mostraron una responsabilidad significativamente superior al resto.

En ambas comunidades la responsabilidad fue significativamente superior en los de menos de 50 años y se elevó significativamente la responsabilidad con el nivel 
escolar. No se manifestó una tendencia clara del tiempo de vida en las comunidades con respecto a la responsabilidad ambiental.

Al identificar los causantes de los problemas ambientales en las encuestas, la mayoría reconocían a todos los factores y a todos los responsables de atender los problemas, lo cual habla a favor de la percepción evaluada. Sin embargo cuando se solicitó información sobre los problemas ambientales que más los afectaban, sólo reflejaban como máximo tres, muy por debajo de la cantidad que existen en esas comunidades (deterioro de la higiene ambiental, degradación de los suelos, falta de educación ambiental, mal manejo de residuos sólidos urbanos y agrícolas, derroche de agua, ruido, contaminación atmosférica y exposición de las personas al sol por un tiempo prolongado).

No existe un reconocimiento mayoritario de los problemas que la comunidad ha tratado o no de resolver. Algunas respuestas manifiestan que ninguno, otros que faltan los recursos, etc.

Actitud ambiental y decisión a incorporarse al cambio

En la Tabla 6.1 aparecen los resultados de la evaluación de la Actitud ambiental de los integrantes de las dos comunidades. (Excelente ó 5, muy bien ó 4, Bien ó 3, Regular ó 2 y mal ó 1). En la tabla 6.2 se expresan las evaluaciones obtenidas por grupos de edad, nivel escolar, tiempo de vida en la comunidad y ocupación, con el correspondiente análisis estadístico.

Tabla 6.1 Número de miembros evaluados en la Actitud ambiental y decisión a incorporarse al cambio

\begin{tabular}{|l|c|c|c|c|c|c|c|c|c|c|}
\hline G. meta & \multicolumn{10}{|c|}{ Evaluación } \\
\hline & \multicolumn{2}{|c|}{ E (5) } & \multicolumn{2}{c|}{ MB (4) } & \multicolumn{2}{c|}{ B (3) } & \multicolumn{2}{c|}{ R (2) } & \multicolumn{2}{c|}{ M (1) } \\
\hline & C.Nvo & CPA & C.Nvo & CPA & C.Nvo & CPA & C.Nvo & CPA & C.Nvo & CPA \\
\hline D & 1 & 0 & 2 & 1 & 10 & 5 & 2 & 1 & 0 & 0 \\
\hline A-J & 2 & 0 & 10 & 2 & 49 & 13 & 52 & 9 & 30 & 14 \\
\hline E & 0 & 0 & 0 & 3 & 35 & 10 & 35 & 3 & 10 & 4 \\
\hline T-C & 2 & 1 & 18 & 8 & 86 & 20 & 69 & 19 & 29 & 10 \\
\hline total & 5 & 1 & 30 & 14 & 180 & 48 & 158 & 32 & 69 & 28 \\
\hline
\end{tabular}


Tabla 6.2 Evaluación de la actitud ambiental y decisión a incorporarse al cambio por grupos y su significación estadística

\begin{tabular}{|c|c|c|c|}
\hline $\begin{array}{l}\text { Variable dependiente } \\
\text { Edad }\end{array}$ & actitud & Medias & Sig. \\
\hline \multirow{3}{*}{ Cacahual } & menos de 35 años & & \\
\hline & Entre 36 y 50 & 1.70 & $a$ \\
\hline & más de 50 & 1.53 & $b$ \\
\hline \multirow[t]{3}{*}{ CPA } & menos de 35 & 1,10 & $b$ \\
\hline & Entre 36 y 50 & 1,50 & a \\
\hline & más de 50 & 1,13 & $b$ \\
\hline \multicolumn{4}{|l|}{ Nivel escolar } \\
\hline \multirow[t]{4}{*}{ Cacahual } & primario & 1.37 & $b$ \\
\hline & secundario & 1.62 & $a$ \\
\hline & preuniversitario & 1.63 & a \\
\hline & universitarios & 1.48 & $a b$ \\
\hline \multirow[t]{4}{*}{ CPA } & primario & 1,14 & NS \\
\hline & secundario & 1,29 & \\
\hline & preuniversitario & 1,28 & \\
\hline & universitarios & 1,33 & \\
\hline \multirow{4}{*}{$\begin{array}{l}\text { Tiempo de vida allí } \\
\text { Cacahual }\end{array}$} & & & \\
\hline & menos de 10 años & 1.31 & $b$ \\
\hline & entre 11 y 20 & 1.52 & $a$ \\
\hline & mas de 20 & 1.62 & $a$ \\
\hline \multirow[t]{3}{*}{ CPA } & menos de 10 años & 1,42 & a \\
\hline & entre 11 y 20 & 1,15 & $b$ \\
\hline & mas de 20 & 1,25 & $a b$ \\
\hline \multirow{9}{*}{ Ocupación Cacahual } & & & \\
\hline & directivos & 1.93 & a \\
\hline & estudiantes & 1.36 & b \\
\hline & trabajadores y campesinos & 1.61 & $b$ \\
\hline & Amas de casa y jubilados & 1.56 & $b$ \\
\hline & directivos & 1,71 & a \\
\hline & estudiantes & 1,10 & $b$ \\
\hline & trabajadores y campesinos & 1,43 & a \\
\hline & Amas de casa y jubilados & 1,00 & $b$ \\
\hline
\end{tabular}

La percepción sobre la actitud ambiental y decisión a incorporarse al cambio fue evaluada de bien o regular para el $65 \%$ de la CPA y de B y R para el $76,5 \%$ de Cacahual. Por grupos meta los mejor evaluados fueron los directivos en la CPA $(71,4 \%)$, seguidos de los trabajadores y campesinos $(67,2 \%)$, amas de casa y jubilados $(57,8 \%)$ y estudiantes (65\%). En Cacahual fueron los directivos $(66 \% \mathrm{~B})$, los estudiantes $(87,5 \%$ de $B$ y R), seguidos por los trabajadores (76\%), las amas de casa $(70,6 \%)$. Como tendencia las amas de casa mejor evaluadas fueron las de más de 50 años de edad y que llevan viviendo allí más de 20 años. Resulta importante que las amas de casa y jubilados de menos nivel escolar, aunque tienen una visión más estrecha, tienen una percepción de la actitud y decisión a incorporarse al cambio superior.

Si se hace un análisis estadístico de estos resultados los directivos de Cacahual y los directivos como los trabajadores y campesinos en la CPA tuvieron una actitud Significativamente superior al resto de los grupos ocupacionales. 
Tanto en Cacahual como en la CPA, la población de 36 a 50 años manifestó una actitud ambiental significativamente superior al resto de los grupos de edades. En Cacahual los que llevan más de 10 años viviendo en la comunidad tuvieron una actitud significativamente superior a los que llevan poco tiempo en ella, pero en la CPA no hubo una tendencia clara en esto. En la CPA el nivel escolar no influyó significativamente en la actitud. En Cacahual los de nivel escolar superior a primaria tuvieron una actitud significativamente superior.

En las encuestas todos reconocen que su medio ambiente debe ser cuidado pero la explicación es estrecha, señalando la mayoría que para elevar la calidad de vida, la salud de la comunidad, etc. La mayoría considera importante la recolección de basura separada, pero que sólo a veces lo hace en su casa, escuela o trabajo.

Al responder sobre la manera en que puede contribuir a la conservación del MA en su hogar, escuela, trabajo o comunidad, las respuestas fueron muy estrechas y decían prácticamente lo mismo.

Otro elemento importante fue que no hubo correspondencia entre los problemas ambientales reconocidos en las primeras preguntas de la encuesta, la existencia de ellos en la comunidad y la referencia a cómo pueden atenuarse desde su lugar de estancia, lo cual muestra inconsistencia en los conocimientos.

\section{Formación medioambiental}

En la Tabla 7.1 aparecen los resultados de la evaluación de la percepción sobre la Formación medioambiental que han recibido los integrantes de las dos comunidades. (Excelente ó 5, muy bien ó 4, Bien ó 3, Regular ó 2 y mal ó 1). En la tabla 7.2 se expresan las evaluaciones obtenidas por grupos de edad, nivel escolar, tiempo de vida en la comunidad y ocupación, con el correspondiente análisis estadístico.

Tabla 7.1 Número de miembros evaluados en Formación medioambiental de los miembros de las comunidades

\begin{tabular}{|l|c|c|c|c|c|c|c|c|c|c|}
\hline G. meta & \multicolumn{10}{|c|}{ Evaluación } \\
\hline & \multicolumn{2}{|c|}{ E (5) } & \multicolumn{2}{c|}{ MB (4) } & \multicolumn{2}{c|}{ B (3) } & \multicolumn{2}{c|}{ R (2) } & \multicolumn{2}{c|}{ M (1) } \\
\hline & C.Nvo & CPA & C.Nvo & CPA & C.Nvo & CPA & C.Nvo & CPA & C.Nvo & CPA \\
\hline D & 1 & 2 & 5 & 2 & 6 & 3 & 2 & 0 & 1 & 0 \\
\hline A-J & 21 & 4 & 50 & 15 & 44 & 14 & 15 & 1 & 13 & 4 \\
\hline E & 21 & 0 & 12 & 3 & 28 & 10 & 16 & 2 & 3 & 5 \\
\hline T-C & 9 & 9 & 78 & 22 & 58 & 14 & 40 & 6 & 19 & 7 \\
\hline total & 52 & 15 & 145 & 42 & 136 & 41 & 73 & 9 & 36 & 16 \\
\hline
\end{tabular}


Tabla 7.2 Evaluación de la Formación medioambiental por grupos y su significación estadística

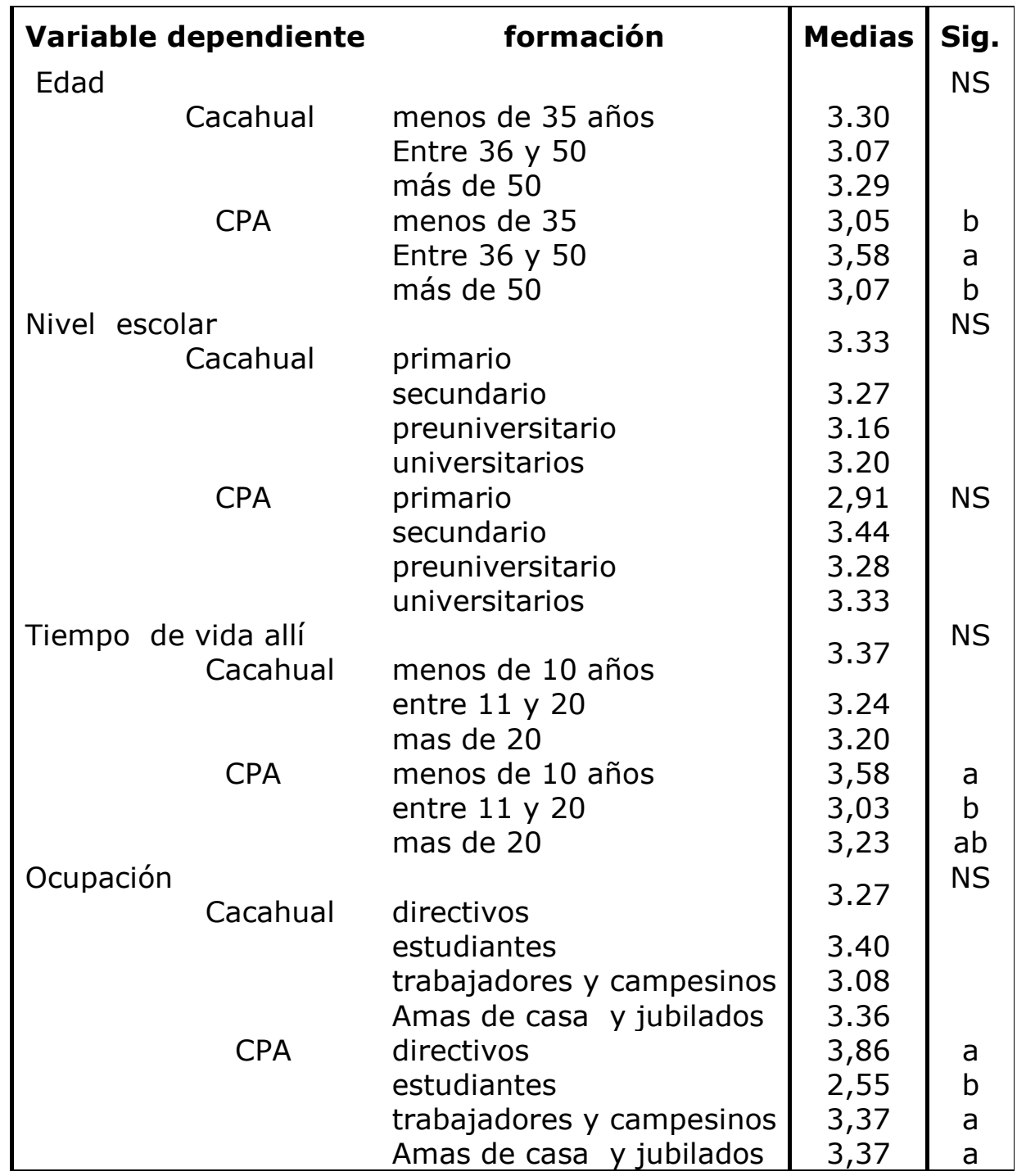

La percepción sobre la formación medioambiental que han recibido fue favorable en las dos comunidades. La mayoría de los miembros la evaluó de E a B (76,3\%). Por grupos meta se observó que todos los directivos la evalúan de E a B en la CPA y el $73 \%$ en Cacahual, el $86,8 \%$ y el $80,4 \%$ de las amas de casa y jubilados y el $77,6 \%$ y $71.07 \%$ de los trabajadores y campesinos en la CPA y Cacahual Nuevo, respectivamente.

En el caso de los estudiantes el 65\% de la CPA evaluó la formación recibida de E a Bien, y en Cacahual el $76,2 \%$. En el grupo de amas de casa y jubilados los de mejores evaluaciones fueron los de mayor edad y más tiempo viviendo en la comunidad. En los estudiantes, los de pre y los universitarios.

Si se hace un análisis estadístico de estos resultados la ocupación no influyó significativamente en la formación ambiental en Cacahual y En la CPA fue inferior significativamente en los estudiantes. La edad no influyó en la percepción sobre la formación medioambiental en Cacahual, pero en la CPA en el grupo 35 a 50 fue significativamente superior. No hubo diferencias significativas para la formación ambiental entre los niveles escolares en ninguna de las dos comunidades. El tiempo de vida en la comunidad no influyó significativamente en la formación medioambiental en Cacahual y en la CPA fue inferior para los que llevan entre 10 y 20 años. 
La mayoría está interesada en aprender sobre medio ambiente, considera que la comunidad ayuda a esto y que la educación ambiental recibida se ha relacionado con la actividad que realiza. Las vías más empleadas para ello son la TV, radio y en algunos casos la prensa. Los directivos y trabajadores reconocen también los cursos.

Pérez y Sánchez (2006:11) evaluando la percepción ambiental en comunidades habaneras detectó una fuerte presencia de la percepción ambiental en la población residente que apunta hacia conocimientos del medio ambiente y a la capacidad de identificación de conflictos ambientales en el entorno, de gran importancia para una participación consciente ante la solución de los problemas, no obstante, existió falta de Educación Ambiental.

\section{CONCLUSIONES}

1- En las comunidades evaluadas existen insuficiencias en la percepción ambiental de sus pobladores. La variable más deficiente resultó ser la percepción acerca de la responsabilidad ambiental y la más favorecida la percepción sobre la formación ambiental que han recibido.

2- La visión ambiental fue evaluada en general entre estrecha y media, significativamente superior para los de nivel universitario y los directivos de Cacahual y no tuvo influencia con la edad ni el tiempo de vida en la comunidad.

3- La responsabilidad ambiental fue evaluada en general entre regular y bien, significativamente superior para los de menor edad, mayor nivel educacional y los directivos.

4- La actitud ambiental y decisión a incorporarse al cambio fue evaluada en general de mal, aunque significativamente superior en los directivos, de mayor nivel universitario de Cacahual y de edad entre 36 y 50 años.

5- La formación medioambiental fue evaluada entre bien y muy bien, sin diferencias significativas en la mayoría de los casos.

6- En las encuestas de ambas comunidades hay falta de correspondencia entre el reconocimiento de los problemas ambientales y la ejemplificación de los mismos en las comunidades, lo cual indica la necesidad de una educación ambiental para lograr su acción con conocimiento de causa.

\section{BIBLIOGRAFÍA}

ALEA, Alina (2005) "Introducción a la psicología ambiental". monografias.com: 9p

BARRAZA M. A. (2007) "Apuntes sobre Metodología de la Investigación. La consulta a expertos como estrategia para la recolección de evidencias de validez basadas en el contenido". INED No. 7. Universidad Pedagógica de Durango. 10p

CITMA, (1997) "Estrategia ambiental Nacional 2006- 2010".Ministerio de Ciencia, tecnología y medioambiente. 67p

COLMENARES E. (2001) "Percepciones Ambientales". Monografías .com. 17p

"Elementos Básicos de percepción ambiental". En línea en http://pmid.proves.ub.edu/becari/psicamb/uni2/2221.htm . Revisado el 28/10/2008

CIGEA "Percepciones medioambientales en la sociedad cubana actual". Un estudio exploratorio (1999) en línea http://www.cuba.cu/ciencia/CIGEA/percepcion.htm Revisado el 28 de octubre 2008. 3p

PÉREZ Norma y Sánchez Carmen Julia (2006) "Medio ambiente y percepción de la población en áreas del parque metropolitano de La Habana: el caso de los consejos populares del municipio Plaza de la Revolución, Cuba" MAPPING Revista Internacional de Ciencias de la Tierra Octubre-nov.http://www.mappinginteractivo.com/plantillaante.asp?id_articulo $=1352$ revisado $28 / 10 / 08$. $13 p$ 
Anexo 1

VALIDEZ DE ACUERDO CON EXPERTOS

Criterio de representatividad de la Percepción ambiental y sus variables
\begin{tabular}{|l|l|l|}
\hline Variables & media & validez \\
\hline Visión del Medio ambiente & 2,71 & fuerte \\
\hline Responsabilidad ambiental & 2,71 & fuerte \\
\hline Actitud ambiental y decisión a incorporarse al cambio & 3,0 & fuerte \\
\hline Formación medioambiental & 2,57 & fuerte \\
\hline
\end{tabular}

Criterio de coherencia de la Visión ambiental, definición, dimensiones, ítems y nivel de medición de los ítems

\begin{tabular}{|l|l|l|}
\hline Variables & media & validez \\
\hline Percepción y visión & 3.0 & fuerte \\
\hline Visión y definición & 2.71 & fuerte \\
\hline Visión y dimensiones & 3.0 & fuerte \\
\hline Visión e items & 2.71 & fuerte \\
\hline $\begin{array}{l}\text { Visión y criterios de } \\
\text { evaluación }\end{array}$ & 3.0 & fuerte \\
\hline
\end{tabular}

Criterio de coherencia de la Actitud, definición, dimensiones, ítems y nivel de medición de los items

\begin{tabular}{|l|l|l|}
\hline Variables & $\begin{array}{l}\text { medi } \\
\text { a }\end{array}$ & validez \\
\hline Percepción y actitud & 3.0 & fuerte \\
\hline Actitud y definición & 2.85 & fuerte \\
\hline Actitud y dimensiones & 3.0 & fuerte \\
\hline Actitud e items & 2.85 & fuerte \\
\hline $\begin{array}{l}\text { Actitud y criterios de } \\
\text { evaluación }\end{array}$ & 2.71 & fuerte \\
\hline
\end{tabular}

Criterio de coherencia de la Responsabilidad, definición, dimensiones, ítems y nivel de medición de los ítems

\begin{tabular}{|l|l|l|}
\hline Variables & media & validez \\
\hline Percepción y Responsabilidad & 2.71 & fuerte \\
\hline Responsabilidad y definición & 2.71 & fuerte \\
\hline Responsabilidad y dimensiones & 2.85 & fuerte \\
\hline Responsabilidad e items & 2.85 & fuerte \\
\hline $\begin{array}{l}\text { Responsabilidad y criterios de } \\
\text { evaluación }\end{array}$ & 2.85 & fuerte \\
\hline
\end{tabular}

Criterio de coherencia de la Formación, definición, dimensiones, ítems y nivel de medición de los ítems

\begin{tabular}{|l|l|l|}
\hline Variables & media & valide \\
\hline Percepción y formación & 2.85 & fuerte \\
\hline Formación y definición & 3.00 & fuerte \\
\hline Formación y dimensiones & 3.00 & fuerte \\
\hline Formación e items & 2.85 & fuerte \\
\hline Formación y criterios de evaluación & 2.85 & fuerte \\
\hline
\end{tabular}




\section{Criterio Calidad técnica de la Percepción ambiental y sus variables}

\begin{tabular}{|l|l|l|}
\hline & media & validez \\
\hline Percepción ambiental & 2.85 & fuerte \\
\hline Visión del Medio ambiente & 2.85 & fuerte \\
\hline Responsabilidad ambiental & 2,57 & fuerte \\
\hline $\begin{array}{l}\text { Actitud ambiental y decisión a incorporarse al } \\
\text { cambio }\end{array}$ & 2.85 & fuerte \\
\hline Formación medioambiental & 2.85 & fuerte \\
\hline
\end{tabular}

\section{ANEXO 2}

\begin{tabular}{|c|c|}
\hline Variable & Intems \\
\hline \multirow[t]{5}{*}{$\begin{array}{l}\text { Visión del medio } \\
\text { ambiente y de los } \\
\text { problemas } \\
\text { ambientales }\end{array}$} & 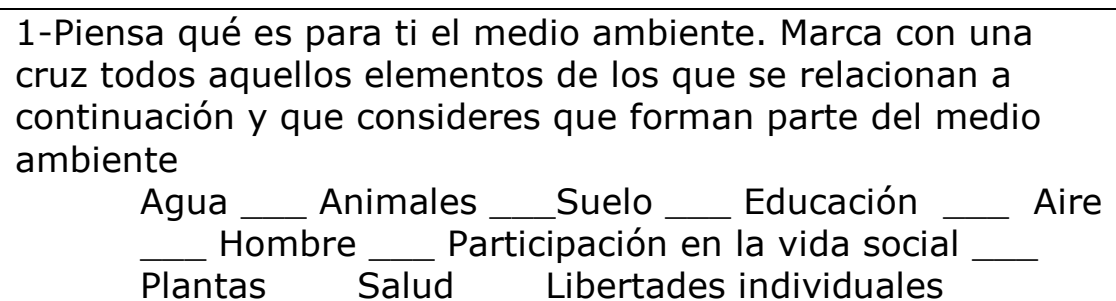 \\
\hline & $\begin{array}{l}\text { 2-Marca con una cruz los elementos que te relacionamos a } \\
\text { continuación que consideras que son problemas ambientales. } \\
\text { Deterioro de la higiene ambiental Degradación de suelos } \\
\text { Pérdida de bosques_Disminución del número de } \\
\text { especies vegetales y animales_Falta de educación } \\
\text { ambiental_Mal manejo de residuos sólidos(basura) y } \\
\text { líquidos agrícolas y urbanos_Agotamiento y contaminación } \\
\text { del agua_ Deterioro de la calidad de vida_ Ruido } \\
\text { Pérdida de patrimonio natural y cultural } \\
\text { Contaminación atmosférica__Calentamiento del planeta }\end{array}$ \\
\hline & $\begin{array}{l}\text { 3-Consideras que tu medio ambiente está afectado de algún } \\
\text { modo. En caso positivo me puedes decir cuáles son los } \\
\text { problemas ambientales que más te afectan. }\end{array}$ \\
\hline & $\begin{array}{l}\text { 4-Con qué extensión se producen los problemas ambientales } \\
\text { En todo el planeta_En una gran parte del planeta_En una } \\
\text { parte del planeta_En una pequeña parte del planeta__No } \\
\text { existen problemas ambientales }\end{array}$ \\
\hline & $\begin{array}{l}\text { 5-Qué importancia le atribuyes a los problemas ambientales } \\
\text { No tienen mucha importancia } \quad \text { Es un tema que está de } \\
\text { moda } \quad \text { Se ha exagerado demasiado } \\
\text { inminente } \quad \text { Puede ser un peligro pero hoy no lo es }\end{array}$ \\
\hline $\begin{array}{l}\text { Responsabilidad } \\
\text { ambiental }\end{array}$ & 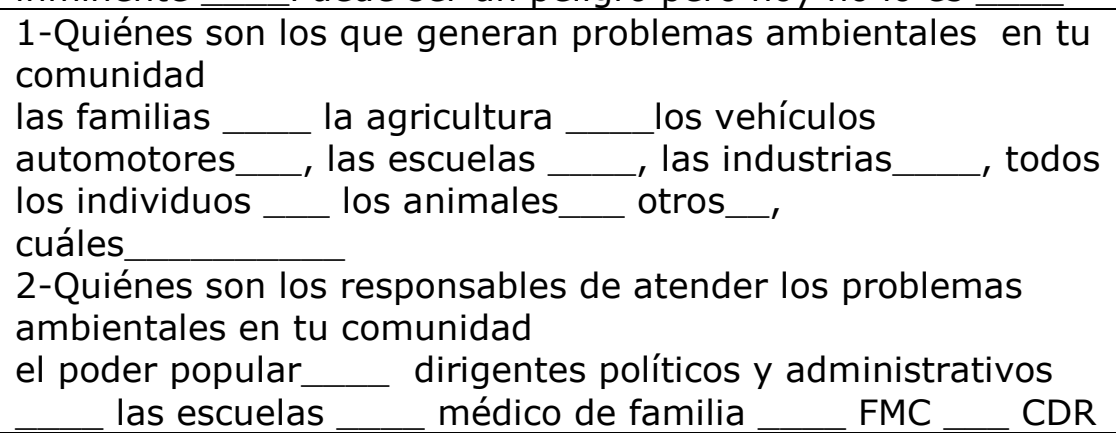 \\
\hline
\end{tabular}




\begin{tabular}{|c|c|}
\hline & $\begin{array}{l}\text { medios de comunicación } \ldots \text { las instituciones } \\
\text { todos los individuos ___ otras__, cuáles }\end{array}$ \\
\hline & $\begin{array}{l}\text { 3-De los problemas ambientales que existen en tu comunidad } \\
\text { cuáles se han tratado de resolver }\end{array}$ \\
\hline & $\begin{array}{l}\text { 4-De los problemas ambientales que existen en tu comunidad } \\
\text { cuáles consideras que no se han tratado de resolver }\end{array}$ \\
\hline $\begin{array}{l}\text { Actitud ambiental y } \\
\text { decisión a } \\
\text { incorporarse al } \\
\text { cambio }\end{array}$ & 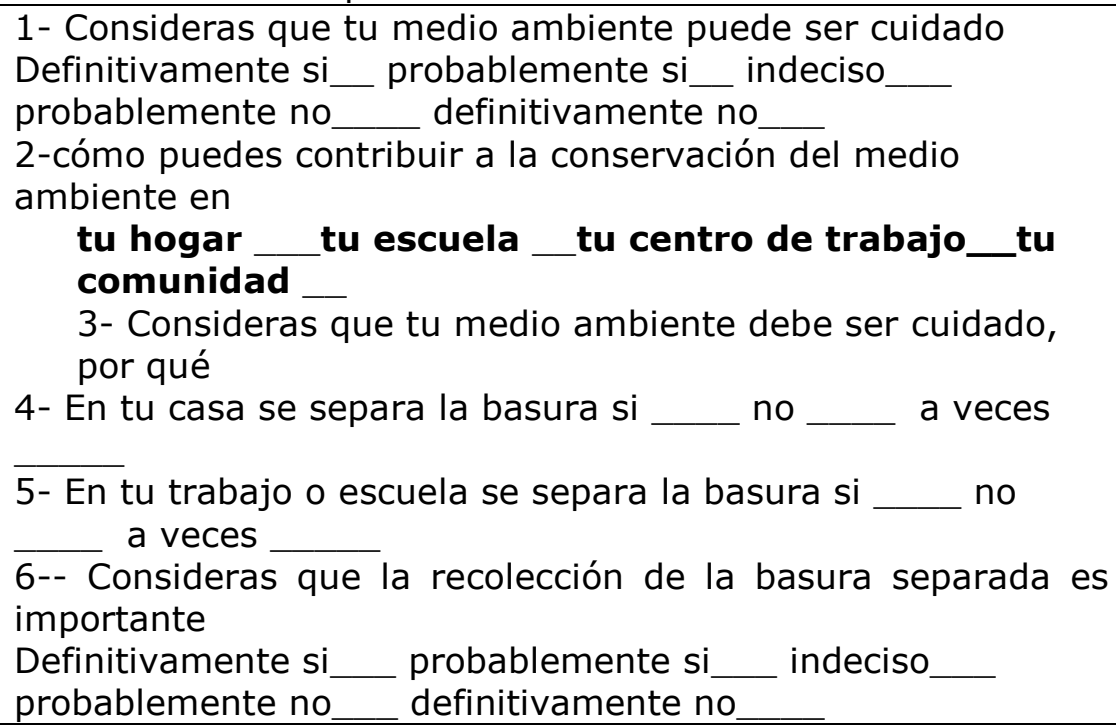 \\
\hline $\begin{array}{l}\text { Formación } \\
\text { medioambiental }\end{array}$ & 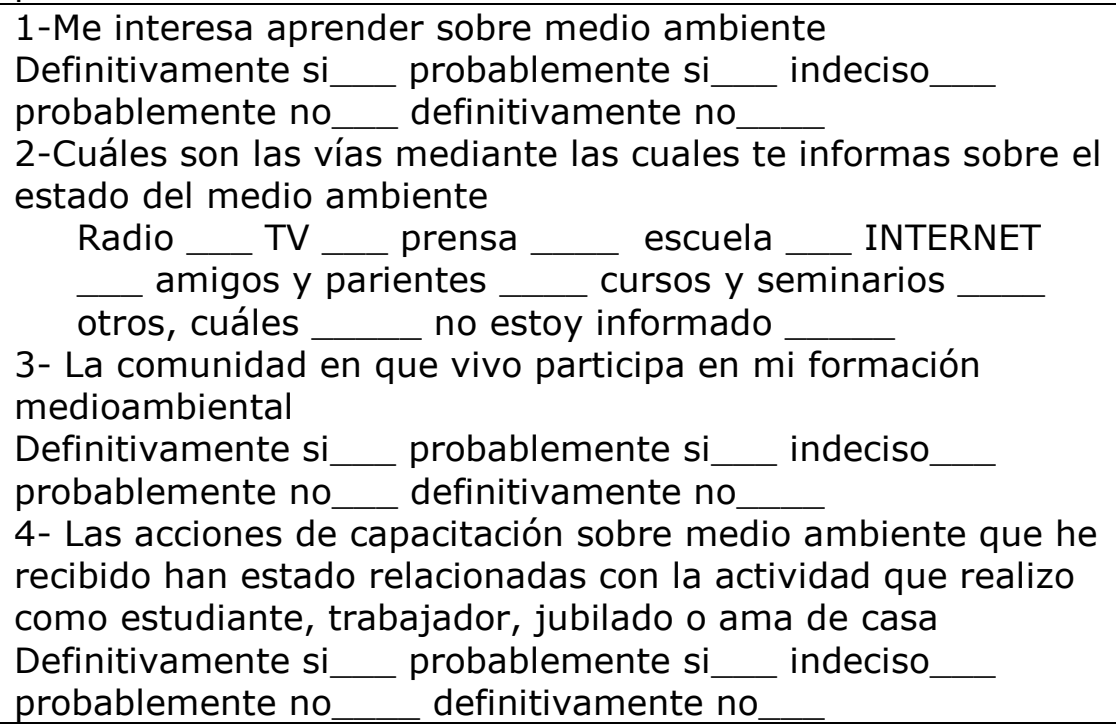 \\
\hline
\end{tabular}

